\title{
The B chromosomes of Pycnogaster cucullata (Charp.) and their effects on the $A$ complement
}

\author{
C. Sentis and \\ J. Fernandez-Piqueras
}

\begin{abstract}
Departamento de Genética, Facultad de Ciencias, Universidad Autónoma de Madrid, Mód, C-XV. Cantoblanco, Madrid-28049, Spain.
\end{abstract}

Pycnogaster cucullata (Charp.) is a polytypic species of Tettigonioidea having XO and XY chromosomal races. In all populations of these races examined, several individuals were found carrying $B$ chromosomes that may be of two morphological types, namely $S t$ and $d$. Although during diplotene the Bs appear almost completely condensed (positively heteropycnotic), and meiotic and mitotic metaphases only two grey intercalary C-bands can be shown. The utilisation of a silver impregnation method allowed us to distinguish the B-chromatin from the facultative heterochromatin of the neo $X$ chromosomes, because both types of heterochromatin present a different condensation cycle during meiotic prophase. The presence of two Bs in nine individuals from the PEG population can be correlated with the simultaneous occurrence of several types of abnormalities affecting the A-chromosomes: spontaneous breakage, depression in the amount of effective pairing, germ line polysomy, endopolyploidy and micronuclei. These effects have also been observed in the individuals of PEG and another population carrying only one B but at a low frequency. While germ line polysomy may be essentially related to the presence and number of Bs (additive effect), determinant factors present in the genetic background cannot be excluded as contributing to the control of effective pairing.

\section{INTRODUCTION}

The species Pycnogaster cucullata (Tettigonioidea, Orthoptera) has been analised in two previous papers (Fernández-Piqueras et al., 1982a; 1983). From these works it was concluded that one B chromosome is present in some individuals of the two main cytological races found in this species. Only the B chromosome of the Truchas ( T) population $\left(X Y_{1}\right.$ race) increased the mean cell chiasma frequency. The decrease between cell variance for chiasma frequency suggested a stabilising role for the $\mathrm{B}$ chromosomes in this population.

A new sample collected from populations of the $\mathrm{XO}, \mathrm{XY}_{1}$ and $\mathrm{XY} \mathrm{Y}_{2}$ races has allowed us to demonstrate the existence of two different types of B chromosomes, and to carry out a more detailed study about the condensation cycle of Bs and their possible influence on the members of the A-complement.
MATERIAL AND METHODS

The individuals studied in this work have been obtained from populations belonging to the previously defined three cytological races of Pycnogaster cucullata (Fernandez-Piqueras et al., 1983). Samples were collected at Sierra de Gredos ( $\mathrm{P}$ and $\mathrm{JH}$ populations, $\mathrm{XO}$ race), León (T population, $X Y_{1}$ race) and Sierra de Guadarrama (MI and $\mathrm{PEG}$ populations, $\mathrm{XY}_{2}$ race) (see table 1).

Standard air dried preparations were obtained and conventional acetic orcein method as well as C-banding (Sumner, 1972) were employed.

The silver impregnation was essentially performed as described by Fernández-Piqueras et al. $(1982 b) ; 1 \mathrm{~g}$ of $\mathrm{AgNO}_{3}$ was dissolved in $1 \mathrm{ml}$ of a sodium citrate solution $\left(10^{-3}\right.$ to $\left.10^{-4} \mathrm{M}\right)$ that had been adjusted to $p \mathrm{H} \mathrm{3-3.5}$ with formic acid. A drop of this solution was placed on the slide and incubated at $60^{\circ} \mathrm{C}$ in a moist chamber for variable 
Table 1 A summary of the effects produced by the presence of B chromosomes on chromosomal instability in the A-complement during spermatogonial and meiotic divisions. 1, spontaneous breakage during meiosis-I. 2, depression of effective pairing estimated by the presence of rod-shaped $L_{1}$ bivalents or univalents (especially $L_{1}$, neo X and neo Y). 3, polysomic cells. 4 , endopolyploid cells. 5 , presence of micronuclei associated with early spermatids. The symbols $(+)$ and $(-)$ refer to the frequences observed for each type of abnormalities. (-), lower than 1 per cent. $(+)$, between 1 and 10 per cent $(++)$, between 10 and 20 per cent. $(++t)$, between 20 and 40 per cent. These frequences have been estimated from a minimum of 100 cells examined for each individual. In the column 5 (micronuclei) the symbols $(+)$ and $(-)$ only denote presence or absence, respectively

\begin{tabular}{|c|c|c|c|c|c|c|c|c|c|}
\hline Populations & $\begin{array}{l}\text { Cytol. } \\
\text { race }\end{array}$ & B-number & B-type & $\begin{array}{l}\text { Individual } \\
\text { number }\end{array}$ & 1 & 2 & 3 & 4 & 5 \\
\hline \multirow[t]{2}{*}{$\mathrm{T}$} & \multirow[t]{2}{*}{$X Y_{1}$} & 0 & & 80 & - & - & - & - & - \\
\hline & & 1 & $S t$ & 4 & - & - & - & - & - \\
\hline \multirow[t]{3}{*}{ PEG } & \multirow[t]{3}{*}{$\mathrm{XY}_{2}$} & 0 & & 6 & - & - & - & - & - \\
\hline & & 1 & $S t$ or $d$ & 7 & + & + & + & - & - \\
\hline & & 2 & St or $d$ & 9 & $+t$ & $+t+$ & $+t+$ & + & + \\
\hline \multirow[t]{2}{*}{ MI } & \multirow[t]{2}{*}{$X Y_{2}$} & 0 & & 10 & - & - & - & - & - \\
\hline & & 1 & $S t$ or $d$ & 2 & - & + & - & - & - \\
\hline \multirow[t]{2}{*}{$P$} & \multirow[t]{2}{*}{ XO } & 0 & & 33 & & - & - & - & - \\
\hline & & 1 & $S t$ or $d$ & 9 & - & - & - & - & - \\
\hline \multirow[t]{2}{*}{$\mathrm{JH}$} & \multirow[t]{2}{*}{$\mathrm{XO}$} & 0 & & 15 & - & - & - & - & - \\
\hline & & 1 & $S t$ & 3 & - & + & - & - & - \\
\hline
\end{tabular}

times. A pretreatment of the slides in 2XSSC at $60^{\circ} \mathrm{C}$ for $10-30$ minutes has allowed us to obtain a clear definition of chromatid axial structures as has been previously shown (Sentis et al., 1984).

\section{RESULTS}

In all examined populations several individuals were found carrying $\mathrm{B}$ chromosomes of two different types (table 1). The most frequent type, named "standard" (St), is a telocentric medium sized chromosome with a proximal euchromatic segment (fig 1(a)). The second type, named "deleted" $(d)$, also is a medium telocentric chromosome, but clearly smaller than the former (fig 1(b)). It is of some interest to note that although most cells of the same individual present a fixed number of Bs, some interfollicular variation has been observed. In the individuals with two Bs the most common situation is that Bs pair effectively and form one proximal chiasma (fig. 1(c)), although when the Bs are of different type do not (fig. 1(b)). Exceptionally ring-shaped $B_{S t}$-bivalents can be observed (fig. 1(d)),

During diplotene the Bs appeared positively heteropycnotic, except for the euchromatic segments mentioned above, but at meiotic and mitotic metaphases they show only two grey intercalary C-bands which allow us to distinguish the Bs from the members of the normal complement (fig. 1(c)).

The silver method after 2 XSSC pretreatment stains differentially the facultative hetero- chromatin of $\mathrm{X}$ and neo $\mathrm{X}\left(X_{L}\right.$ limb) during the most condensed stages of meiosis (FernándezPiqueras et al., 1982b). Nevertheless, with this method, the B chromosomes found in p. cucullata display the same degree of staining that the autosomes at those stages (fig. 1(f)). In addition, after silver staining the sex bivalents from the $X Y$ races show one darkly stained nucleolus attached to the nucleolar organising region (NOR) (FernándezPiqueras et al., 1983). This feature has allowed us to distinguish the $\mathrm{B}$ chromatin from the facultative heterochromatin of the $X_{I}$, region of the neo $X$ chromosome (primitive $\mathrm{X}$ chromosome in XO system) during first meiotic prophase. A detailed observation shows that both types of heterochromatin present a different condensation cycle since at early prophase only the $X_{L}$-chromatin becomes condensed, while B-chromatin is almost indistinguishable from the chromatin of the normal complement (fig. 1(g)). At a later stage the Bchromatin starts to become more evident (fig. 1(h)) and, finally, at pachytene both types of chromatin exhibit a similar degree of staining and condensation (fig. 1(i)).

Although the A-complement usually remains unaffected by the presence of $\mathrm{Bs}$, the presence of two Bs in nine individuals from the PEG population could be correlated with the simultaneous occurrence of several types of abnormalities: breakage (fig. 2(a)), depression in the amount of effective pairing (figs. 2(b) and (c)), germ line polysomy (figs. 2(b) and (d)), endopoliploidy (figs. $2(\mathrm{e})$ and (f)), and the subsequent appearance of 

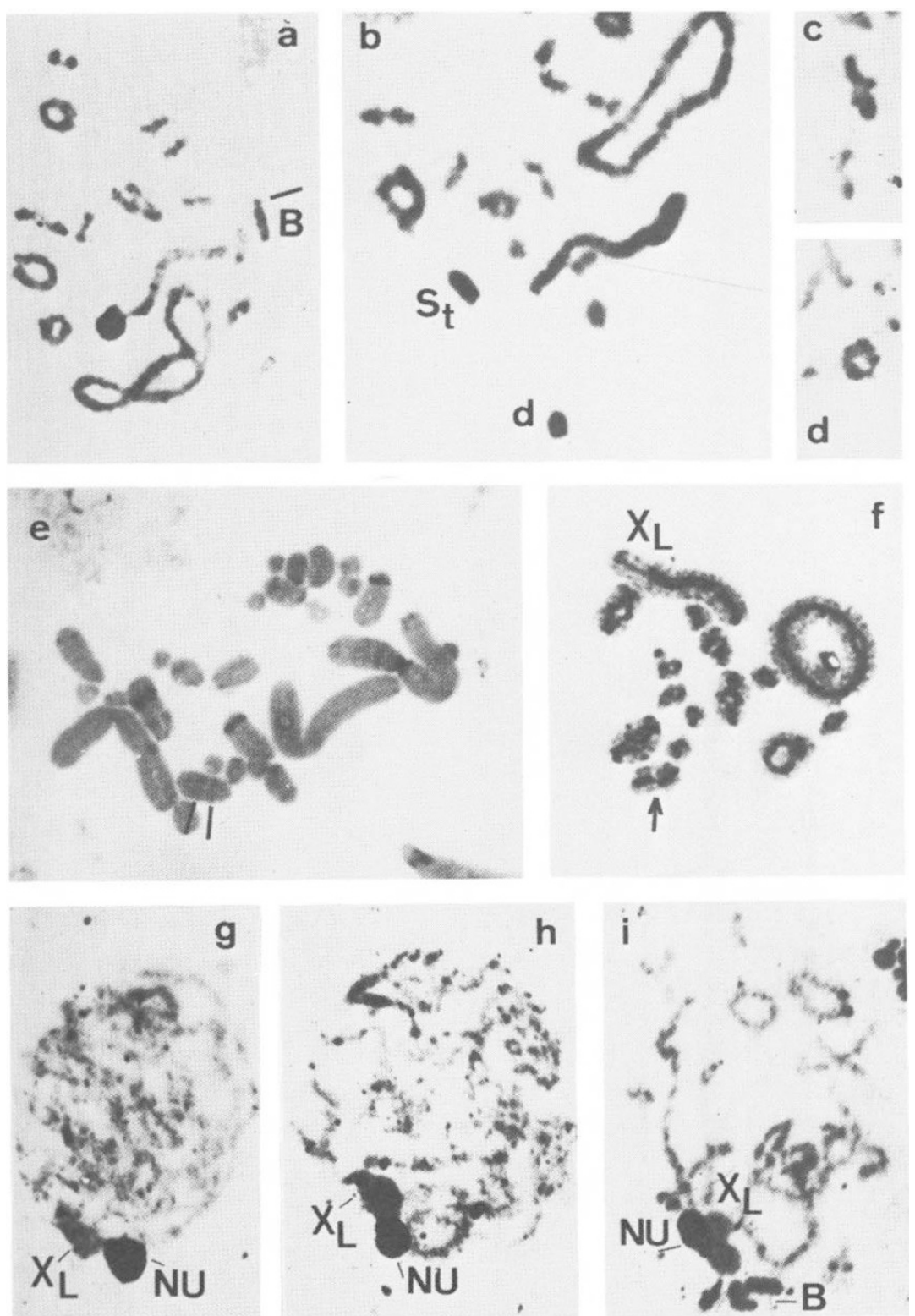

Figure 1 Structure of the B chromosomes found in $P$. cucullata. (a) Diplotene cell of an individual from the T population carrying one Standard B chromosome (B). Note a proximal euchromatic segment on this chromosome (line). (b) Idem from another individual of the PEG population with two Bs (univalents) of different type ( $S t$ and deleted, $d$ ). (c) Homozygous B-bivalent (St-St) with a proximal chiasma. (d) Ring $\mathrm{B}_{S t}$ bivalent. (e) C-mitotic metaphase from an individual of the PEG population carrying one $\mathrm{B}_{\mathrm{St}}$-chromosome. Note two intercalary grey C-bands on this chromosome (lines). (f) Differential (negative) staining of the $\mathrm{X}_{L}$ segment of the neo $\mathrm{XY}$ sex bivalent during metaphase I after silver impregnation. Note that the B-bivalent (arrow) stains similar to the autosomes. (g), (h) and (i) Three different stages during prophase I after silver impregnation. NU, nucleolus. $\mathrm{B}, \mathrm{B}$-bivalent. $\mathrm{X}_{L}$, long-arm of the neo $\mathrm{X}$ chromosome (primitive $\mathrm{X}$ at $\mathrm{XO}$ stage) consisting of facultative heterochromatin.

micronuclei associated with early spermatids (fig. $2(\mathrm{~g})$ ). Some of these phenomena have also been observed, but less frequently, in individuals carrying only one $\mathrm{B}$ from the $\mathrm{JH}, \mathrm{MI}$ and $\mathrm{PEG}$ populations (see table 1). The depression in the amount of effective pairing, which results in the frequent appearance of rod-shaped bivalents as well as univalents at first meiosis, preferentially affects the $L_{1}$ and neo XY bivalents (figs. 2(b) and (c)). Nevertheless, polysomy has only been observed 

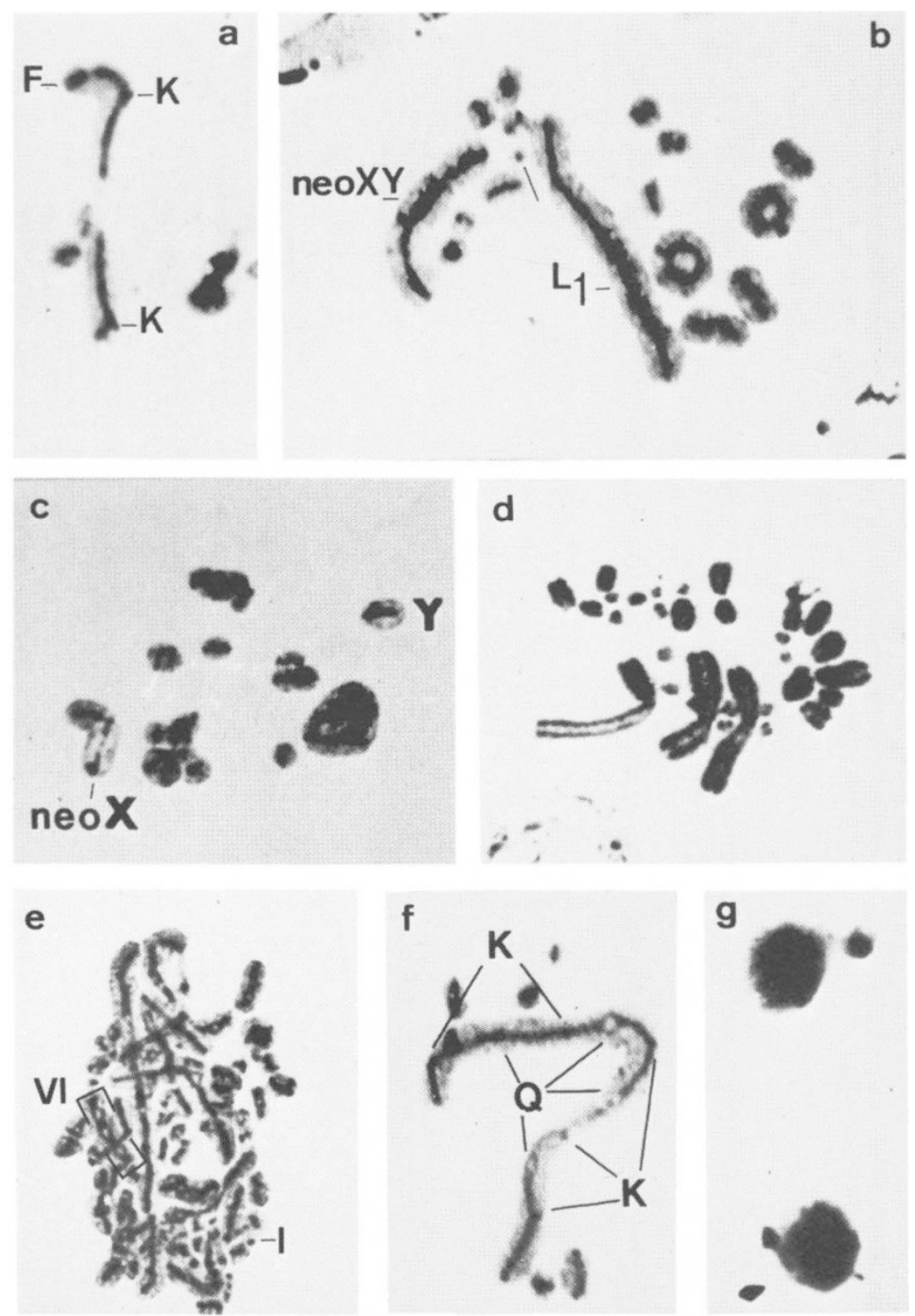

Figure 2 Chromosomal instability. (a) Breakage affecting one $L_{1}$ bivalent. The fragment (F) remains attached by a terminalised chiasma (arrow). K, kinetochores. (b) Metaphase I of one individual from the PEG population that usually carries two Bs, showing only one B and three extra short-chromosomes as one bivalent and one univalent (line). Decrease of effective pairing results in a rod-shaped $L_{1}$ bivalent. (c) Idem showing the sex chromosomes, neo X and neo Y, as univalents. (d) Spermatogonial metaphase nucleus carrying two extra short chromosomes (polysomy) and two Bs. The extra copies cannot be reliably identified because all short chromosomes are of similar length. (e) Endopolyploid cell at metaphase I showing different types of configurations from hexavalents (VI) to univalents (I). These features suggest that this cell was previously polysomic. (f) Pentavalent $L_{1}$ configuration from an endopolyploid metaphase I cell. K, kinetochores. Q, chiasmata. (g) Early spermatids with associated micronuclei.

for the short chromosomes ( $S$-type), and the cells of a given follicle can show a variable number of extra copies (from 1 to 6) (figs. 2(b) and (d)). Monosomic or nulisomic cells have not been observed.
One additional point that needs some comment is the fact that no differences have been observed when either standard of deleted B chromosomes were present. 


\section{DISCUSSION}

Two of the questions which still preoccupy those who study B chromosomes are the nature of their chromatin and the possible influence of Bs, together with the genetic background, on the chromosome behaviour of the A chromosomes.

\section{(i) B-heterochromatin}

Although the majority of B chromosomes in animals have been described as heterochromatics, the development of C-banding to identify constitutive heterochromatin has demonstrated that the amount of heterocrhomatin in Bs described as heterochromatics varies substantially (Jones and Rees, 1982). In the species Picea glauca, for example, the Bs form chromocentres at interphase but Giemsa staining does not show constitutive heterochromatin (Teoh and Rees, 1977). This capacity for heterochromatinisation at interphase has been considered by several authors to be a facultative process similar to that of the X chromosome. The Bs of $P$. cucullata neither form chromocentres at interphase nor present considerable C-positive regions at metaphase (fig. 1(e)), being positively heteropycnotic only during pachytene and diplotene (figs. 1(a), (b) and (i)). At first sight most parts of B-chromatin and the chromatin of the sex chromosomes seem to be of the same facultative kind, but a comparative analysis during first meiotic prophase clearly reveals differences in the condensation cycles of both types of chromatin (figs. 1(g), (h) and (i)). Additionally the differential negative heteropycnosis shown in $\mathrm{X}$ or $\mathrm{X}_{L}$ during the most condensed stages of meiosis, cannot be observed in the Bs (fig. 1(f)). Thus in $P$. cucullata it is possible to distinguish three different types of heterochromatic regions after silver impregnation and C-banding methods: B-heterochromatin, X-heterochromatin and constitutive C-heterochromatin.

\section{(ii) Chromosomal instability}

B-chromosomes have been described in many orthopteran species but reports about their possible influence modifying the number or structure of the A chromosomes are very rare. On the other hand, some chromosome abnormalities are difficult to interpret precisely when only conventional or C-banding techniques are employed. However, silver staining methods can be used to demonstrate chromatid axial elements during the most condensed stages of mitosis and meiosis. The morphology of these axial structures is completely representative of the shape of the respective chromosomes or bivalents, and includes differentiated regions which coincide with kinetochores, secondary constrictions (including NORs) and chiasmata (Sentís et al., 1984). This may be of great interest in chromosome characterisation and could be used to identify cryptic chromosome rearrangements. Using this method we have found evidence of spontaneous chromosome breakage in some meiotic chromosomes, especially in those individuals of $P$. cucullata carrying two Bs. The fragments produced by breakage usually remain attached by chiasmata (fig. 2(a)). The rarity of free fragments at metaphase-I suggests that breakage is a post-pairing event. A similar case has been described in Trillium grandiflorum where the presence of B chromosomes has already been correlated with spontaneous breakage in the As (see Jones and Rees, 1982).

It has been shown many times that Bs influence the amount and distribution of chiasmata in the A complement (Hewitt, 1979; Jones and Rees, 1982). Furthermore in some populations of Lolium Bs seem to depress the amount of effective pairing and chiasma formation, not only between homologues but also more drastically between homeologous chromosomes in hybrids (Evans and Macefield, 1972; 1973 and 1974). This suggests that determinant factors on both As and Bs effectively control chromosome pairing. The genotypedependent effects of Bs on chiasma frequency has also been shown by Henriques-Gil et al. (1982).

The effect of the B chromosomes of $P$. cucullata on pairing or chiasma formation, while it depends on the number of Bs, also varies from one population to another. Thus, while individuals from the $\mathrm{T}$ population show an increase in the mean cell chiasma frequency, this effect was not observed in individuals carrying one $\mathrm{B}$ from the $\mathrm{P}$ population (Fernández-Piqueras et al., 1982a). Individuals with two Bs from the PEG population display, however, a significant reduction in chiasma formation (especially affecting the $L_{1}$ and neo XY bivalents) including a high level of univalents (fig. $2 c$ ). The individuals with only one $B$ from the PEG, $\mathrm{JH}$ and MI populations show a reduction in effective pairing, but this effect is more enhanced in those individuals carrying two Bs in the PEG population. Finally, this anomaly has not been found in those individuals with one $\mathrm{B}$ from the $\mathrm{T}$ and $\mathrm{P}$ populations. In view of these results, it can be concluded that determinant factors in the genetic background cannot be excluded as having an 
influence on effective pairing of the As, in addition to the presence and number of Bs (see table 1).

The numerical variations which have been outlined are also probably influenced by the presence of Bs. A comparable case is described by Hewitt and East (1978), who found polyploids and aneuploids among malformed embryos of Myrmeleotettix maculatus when B chromosomes were present. However, other numerical variations such as germ line polysomy, have been described which cannot be correlated with the presence of Bs (Hewitt and John, 1968; Peters, 1981; Camacho et al., 1981). Germ line polysomy in $P$. cucullata has only been observed in individuals with two Bs from the PEG population (fig. 2(d)), but not in individuals with 0 or $1 \mathrm{~B}$. This seems to indicate that mitotic instability of the As may probably be related not only to the presence but also to the number of Bs, thus resembling the type $A$ additive effect which has been attributed to Bs by several authors (see Jones and Rees, 1982).

\section{REFERENCES}

C:AMACHO, J. P. M., DIAZ DE LA GUARIDIA, R. AND RUIZ REJON, M. 1981. Polysomy and supernumerary isochromosomes in the grasshopper Omocestus bolivari (Chopard). Heredity, 46, 123-126.

EVANS, G. M. AND MACEFIFI. A. J. 1972. Suppression of homeologous paring by $\mathbf{B}$ chromosomes in a Lolium species hybrid. Nature Lond. New Biology, 236, 110-111.

EVANS, G. M. AND MACEFIELI), A. J. 1973. The effect of B chromosomes on homeologous pairing in species hybrids. 1. Lolium tomulentum $\times$ Lolium perenne. Chromosoma (Berl.), 41, 63-71.
EVANS, (;. M. AND MACEFIELD,.A. J. 1974. The effect of B chromosomes on homeologous pairing in species hybrids. II. Lolium multiflorum $\times$ Lolium perenne. Chromosoma (Berl.), 45, 369-378.

FERNANDEZ-PIQUERAS, J., RODRIGUEZ CAMPOS, A., SENTIS CASTAÑO, C. AND WANDOSEll, F. 1982a. Pycnogaster cucullata (Charp.) a polytypic species of Tettigonioidea with $\mathrm{XO}$ and neo XY sex determination. Heredity, 48, 147-150.

FERNANDEZ-PIQUERAS, J., RODRIGUIZ CAMPOS, A., SENTIS CASTAÑO, C. AND ROJO, E. 1982b. Differential staining of the X-chromosome during meiosis of Orthoptera by a silver impregnation procedure. Chromosoma (Berl.), 85, 707-711.

FER NANDEZ-PIQUERAS, J., RODRIGUEZ CAMPOS, A., SENTIS CAstaño, C. AND Rojo, E. 1983. Sex chromosome evolution in the polytypic species Pycnogaster cucullata. Heredity, 50, 217-223.

HENRIQUES-GiL, N., SANTOS, J. L. AND GIRALDEZ, R. 1982. Genotype-dependent effect of B-chromosomes on chiasma frequency in Eyprepocnemis plorans (Acrididae: Orthoptera). Genetica., 59, 223-227.

HEWITT, (;. M. 1979. Grasshoppers and crickets. Animal Cytogenetics. Vol. 3, Insecta 1, Orthoptera. Gebrüder Borntraeger, Berlin-Stuttgart.

HEWITT, G. M. ANI EAST, T. M. 1978. Effects of B chromosomes on development in grasshopper embryos. Heredity, 41, 347-356.

HEWITT, G. M. AND JOHN, B. 1968. Paralled polymorphism for supernumerary segments in Chorthippus parallelus (Zetterstedt). I. British populations. Chromosoma (Berl.), 25, 319342.

JONES, R. N. AND REES, H. 1982. B chromosomes. Academic Press, London, New York.

PETERS, G. B. 1981. Germ line polysomy in the grasshopper Attractomorpha similis. Chromosoma (Berl.), 81, 593-617.

SENTIS, C., RODRIGUEZ CAMPOS, A., STOCKERT, J. C. AND FERNANDEZ-PIQUERAS, J. 1984. Morphology of the axial structures in the neo XY sex bivalent of Pycnogaster cucullata (Orthoptera) by silver impregnation. Chromosoma (Berl.), 90, 317-321.

TEOH, S. B. AND REES, H. 1977. B chromosomes in White Spruce. Proc. Roy. Soc. B., 198, 325-344. 